\title{
Monographie des légumineuses subligneuses utilisées pour la biofertilisation des sols dans les jachères améliorées dans la localité de Daloa (Côte d'Ivoire)
}

\author{
${ }^{1}$ AKEDRIN Tetchi Nicaise, ${ }^{2}$ AKOTTO Odi Faustin, ${ }^{3}$ COULIBALY Kiyinlma, ${ }^{4}$ COULIBALY \\ Siendou, ${ }^{5} \mathrm{AKE}$ Sévérin \\ 1,4Laboratoire d'Amélioration de la Production Agricole, UFR Agroforesterie, Université Jean Lorougnon Guédé, BP 150 Daloa, \\ Côte d'Ivoire ; \\ ${ }^{2}$ Département de la Science du Sol / Phytopedologie / Relation sol-plante ; UFR Science de la Terre des Ressources Minières,

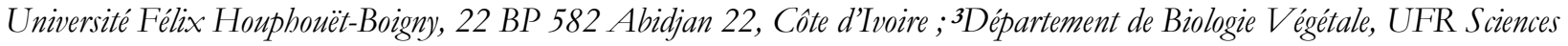 \\ Biologiques, Université Peleforo Gon Coulibaly, BP 1328 Korbogo ; \\ 4Laboratoire de Physiologie Végétale, UFR Biosciences, Université Félix Houphouët-Boigny, 22 BP 582 Abidjan 22, Côte \\ d'Ivoire. \\ Corresponding author e-mail : akedrinick@yahoo.fr Téléphone 00(225) 069011 91;00 (225) 07401135
}

Mots clés : légumineuses herbacées, prospections, fertilité du sol, espèces biofertilisantes, Côte d'Ivoire Key words: herbaceous legumes, surveys, soil fertility, biofertilizing species, Côte d'Ivoire.

Publication date 31/07/2020, http://m.elewa.org/Journals/about-japs/

\section{RÉSUMÉ}

En Côte d'Ivoire, la pratique des cultures continues ont entrainé la pauvreté des sols en éléments nutritifs. Pour y remédier, des espèces biofertilisantes en occurrence des légumineuses subligneuses susceptibles d'influer sur la disponibilité des éléments nutritifs du sol sont plantées dans les jachères pour la restauration de la fertilité. Cette étude sur le recensement et les caractérisations physicochimiques du sol et des légumineuses utilisées comme biofertilisants dans les jachères a été conduite à Daloa. La caractérisation chimique de ces végétaux réalisée sur la biomasse racinaire fraîche séchée au four à $65^{\circ} \mathrm{C}$ pendant 72 heures puis broyée et tamisée à une taille de $0,5 \mathrm{~mm}$, a porté sur les compositions totales en $\mathrm{N}, \mathrm{P}, \mathrm{K}, \mathrm{Ca}, \mathrm{Mg}, \mathrm{C}$. Quant à la caractérisation du sol prélevé à $0-20 \mathrm{~cm}$ de profondeur, le $\mathrm{pH}$, la taille des particules, l'azote total, le carbone organique, la capacité d'échange de cations, le $\mathbf{P}$ disponible et le $\mathrm{K}$ ont été analysés. Les prospections réalisées dans ces jachères ont permis de recenser six légumineuses comme culture principale. Ces légumineuses sont majoritairement lianescentes $(50 \%)$ contre $33 \%$ des herbacées et $17 \%$ d'arbustes. Les caractéristiques chimiques des racines ont montré une différence de variation de l'azote du témoin $\left(13 \mathrm{~g} \mathrm{~kg}^{-1}\right)$ à Mimosa invisa $\left(28 \mathrm{~g} \mathrm{~kg}^{-1}\right)$. Les concentrations en $\mathrm{N}, \mathrm{P}, \mathrm{K}$ ont été maximales chez toutes les espèces à l'exception de Mimosa invisa et Crotalaria retusa. Cependant, chez Mimosa invisa, les fortes concentrations de CEC (13,8 cmolc $\left.\mathrm{kg}^{-1}\right)$ et de C (27 g kg-1) ont été enregistrées. Les concentrations de phosphores $\left(3,9 \mathrm{~g} \mathrm{~kg}^{-1}\right)$ et de potassiums (53 $\mathrm{g} \mathrm{kg}^{-1}$ de $\mathrm{K}$ ) sont maximales respectivement chez Crotalaria goreensis et Crotalaria retusa. L'usage de ces légumineuses semble indispensable à la reconstitution de l'horizon humifère donc susceptible de restaurer la fertilité du sol et d'influencer la croissance des cultures non fixatrice d'azote. 
Monograph of subligneous legumes used for the biofertilization of improved fallow soils in Daloa (Côte d'Ivoire).

\begin{abstract}
In Côte d'Ivoire, the practice of continuous cultivation has resulted in poor nutrient soils. To remedy this, biofertilizing species in the form of subligneous legumes likely to influence the availability of soil nutrients are planted in fallows to restore fertility.

This study on the inventory and the physico-chemical characterization of the legume species used as biofertilizers in fallows was conducted in Daloa. The chemical characterization of these plants was carried out from fresh root biomass dried in an oven at $65^{\circ} \mathrm{C}$ for 72 hours then crushed and sieved to a size of $0.5 \mathrm{~mm}$. The analyses related to the total compositions in $\mathrm{N}, \mathrm{P}, \mathrm{K}, \mathrm{Ca}, \mathrm{Mg}$, and $\mathrm{C}$. As for the characterization of the soil sampled at $\mathbf{0 - 2 0} \mathrm{cm}$ depth, the $\mathrm{pH}$, the size of the particles, Total nitrogen, the organic carbon, cation exchange capacity, available $\mathbf{P}$ and $\mathrm{K}$ were analyzed. Surveys carried out in these fallows have identified six legumes as the main crop. These legumes are mostly creeping $(50 \%)$ compared to herbaceous $(33 \%)$ and shrubs $(17 \%)$. The chemical characteristics of the roots showed a difference in the variation of nitrogen from the control (13 g kg-1) to Mimosa invisa (28 $\mathrm{g} \mathrm{kg}-1)$. Concentrations of $\mathbf{N}, \mathbf{P}$, and $\mathrm{K}$ were highest in all species except Mimosa invisa and Crotalaria retusa. However, in Mimosa invisa, high concentrations of CEC (13.8 cmolc kg-1) and C (27 g kg-1) were recorded. Similarly, the concentrations of phosphorus $(3.9 \mathrm{~g} \mathrm{~kg}-1)$ and potassium $(53 \mathrm{~g}$ kg-1 K) are maximum in Crotalaria goreensis and Crotalaria retusa respectively. The use of these legumes seems essential to the reconstruction of the humiferous horizon, therefore likely to restore soil fertility and influence the growth of crops that do not fix nitrogen.
\end{abstract}

\section{INTRODUCTION}

En Afrique de l'Ouest particulièrement en Côte d'Ivoire, la pratique de la jachère a été favorisée par la grande disponibilité des terres. Cette pratique, basée sur l'alternance d'une phase de cultures continues de 1 à 5 ans, avec une phase de repos du sol qui pouvait durer de 10 à 30 ans, permettait de maintenir et améliorer les rendements des cultures (Floret et Pontanier, 2000 ; Akedrin, 2013). Cependant, depuis des décennies, l'augmentation de la population combinée à une augmentation du cheptel a entraîné une augmentation de la demande en produits végétaux utiles à l'homme et à l'animal (Danso, 1995). Cette forte pression sur le couvert végétal influence négativement la fertilité des sols et cela constitue un obstacle majeur à l'amélioration de la productivité des cultures et à la production de la biomasse nécessaire aux besoins d'une population de plus en plus nombreuse (Bado, 2002). Des efforts visant à atténuer ces problèmes liés à la pauvreté des sols ont exploré plusieurs associations culturales rentables, telles que l'agroforesterie, l'agriculture de conservation et la plantation des espèces biofertisantes à travers le raccourcissement du temps de jachère naturelle. En effet, pour Hien et al. (1993), la jachère courte a besoin d'être améliorée pour répondre à la fois à son double objectif d'augmentation de la production et de restauration de la fertilité des sols ayant atteint un certain niveau de dégradation dans un écosystème fragile. Toutefois, l'utilisation des ressources organiques sur les terres agricoles améliore la fertilité des sols, mais cela reste cruciale pour des zones des tropiques humides et subhumides dominées par des sols à faible capacité d'échange de cations (Partey et al., 2011, Partey et al., 2019). Plusieurs résultats tels que ceux de Beedy et al. (2010), Partey et al. (2011) et de Vanhie et al. (2015) ont confirmé les valeurs d'équivalence en engrais des légumineuses. Dès lors, de nombreuses tentatives se faisant de plus en plus grande, ont visé à développer des itinéraires agricoles 
alternatifs orientés vers les jachères plantées en légumineuses (Peltier et al., 1995). Cependant, seules quelques études, quantifiant le fort potentiel de fixation de l'azote des herbacées vivaces ou subligneuses à partir de la biomasse racinaire (Gardener et al., 2001), présentent des informations relatives aux indicateurs de fertilité du sol, telles que la disponibilité de N. Les informations sur l'influence des accroissements de rendements de cultures (Fournier et al., 2001 ; Carsky et al., 2001) de ces végétaux à partir des

\section{MATERIEL ET METHODES}

3.1 Site d'étude : L'étude a été réalisée dans les jachères agroforestières à Daloa, une région du Centre-Ouest de la Côte d'Ivoire. Ces sites avaient été en friche pendant cinq ans avant la réalisation de cette étude. Daloa est situé entre $06^{\circ} 51^{\prime}$ et $06^{\circ} 59^{\prime}$ de latitude Nord et $06^{\circ} 36$ et $06^{\circ} 44^{\prime}$ de longitude Ouest. Le climat est caractérisé par un régime équatorial et subéquatorial à deux saisons de pluies (AvrilJuillet) et (Septembre-Novembre) et deux saisons sèches (Décembre-Mars) et (Juillet Septembre). La pluviométrie est comprise entre 1000 et $1500 \mathrm{~mm} / \mathrm{an}$ avec des températures variant de $24,65{ }^{\circ} \mathrm{C}$ à $27,75{ }^{\circ} \mathrm{C}$ en moyenne (N’Guessan et al., 2014). La presque totalité du bassin se trouve en zone tropicale humide avec une végétation de forêt dense à évolution régressive. La dégradation de cette forêt est accélérée par l'intensification des cultures de rente (cacao, café, palmier à huile et hévéa). Le type de sol sur les sites de l'étude est un Ferralsol.

3.2 Caractérisation des végétaux : Les espèces recensées ont été : Abrus precatorius (Pois rouge ou liane réglisse), Crotalaria goreensis (Chanvre de Malabar ou Pistache bâtard), Crotalaria retusa (Crotalaire), Clitoria ternatea (Clitorie de ternate ou Pois savane), Indigofera arrecta (Indigotier) et Mimosa invisa (Sensitive géante). La sélection de ces espèces a tenu compte de leur abondance relative dans la zone d'étude, leurs valeurs agronomiques et leurs capacités d'approvisionnement en éléments nutritifs avérées. Au Laboratoire d'Amélioration de la Production Agricole de l'Université Jean Lorougnon Guédé (Daloa), ces spécimens ont paramètres de sol devraient être pertinentes pour suggérer une utilisation dans les systèmes de culture. L'objectif de cette étude est de recenser les espèces biofertilisantes susceptibles d'influer sur la disponibilité des éléments nutritifs du sol plantées dans les jachères améliorées. De façon spécifique, il s'agit de déterminer les caractéristiques non seulement botaniques de ces espèces recensées ainsi que physicochimiques du sol sous ces légumineuses.

été identifiés et leur nom scientifique noté. Pour la nomenclature, les flores de Hutchinson et Dalziel (1954) puis celles de Lebrun et Stork (1997) ont été utilisées. En ce qui concerne la caractérisation chimique de ces végétaux, la biomasse racinaire fraîche de chaque espèce déjà établies sur ces sites d'étude a été collectée. Pour la détermination de la composition chimique des racines, les espèces ont été séchées au four à 65 - C au Laboratoire des Sciences des Sols de l'Université Félix Houphouët-Boigny de Côte d'Ivoire pendant 72 heures puis broyés à l'aide d'un broyeur et tamisés à une taille de $0,5 \mathrm{~mm}$. Les matières végétales tamisées ont été analysées pour déterminer les compositions totales en $\mathrm{N}$, $\mathrm{P}, \mathrm{K}, \mathrm{Ca}, \mathrm{Mg}, \mathrm{C}$, en quatre répétitions. Pour toutes les analyses, l'azote total et le carbone total ont été déterminés simultanément par combustion sèche à l'aide de l'autoanalyseur LECO TruSpecTM CN (LECO Corporation), tandis que le $\mathrm{K}$, le $\mathrm{Ca}$ et le $\mathrm{Mg}$ totaux ont été déterminés par la méthode de spectrophotométrie d'absorption atomique et par incinération décrite par Eneji et al. (2005). Le phosphore a également été déterminé dans une solution de cendres par la méthode au phosphomolybdate d'ammonium (Motsara et Roy, 2008).

3.3 Caractérisation initiale du sol: Avant la collecte des espèces, dix échantillons composites en vrac de cinq sous-échantillons de sol de la jachère ont été prélevés au hasard à 0 $20 \mathrm{~cm}$ de profondeur avec une tarière en diagonale pour la caractérisation. Ils ont ensuite été séchés à l'air et tamisés à une taille de $2 \mathrm{~mm}$ 
avant d'être analysés à l'aide de quatre souséchantillons répliqués à partir de l'échantillon composite. Le $\mathrm{pH}$ du sol a été mesuré avec une électrode en verre (1: $1 \mathrm{H} 2 \mathrm{O})$ et la taille des particules par la méthode de l'aréomètre. L'azote total a été déterminé par combustion sèche en utilisant l'auto-analyseur LECO TruSpecTM CN (LECO Corporation), le carbone organique par la méthode d'oxydation au bichromate (Motsara et Roy 2008), la capacité d'échange de cations par photométrie de flamme avec extrait d'acétate

\section{RÉSULTATS ET DISCUSSION}

4.1 Monographie des espèces recensées : $\mathrm{Au}$ terme de nos investigations, les six espèces recensées se répartissent en cinq espèces de Fabaceae comprenant quatre genres (Abrus, Clitoria, Crotalaria et Indigofera) et une espèce de Mimosaceae comportant un genre (Mimosa).

\subsubsection{Espèces de la famille de Fabaceae} 4.1.1. 1 Abrus Precatorius L. (Fabaceae)

Morphologie : Liane volubile subligneuse, elle peut atteindre $2 \mathrm{~m}$ de hauteur et $5 \mathrm{~cm}$ de circonférence. Les feuilles, composées paripennées, alternes, pétiolées, sont glabrescentes. Les folioles, au nombre de 10 à 20 paires, sont oblongues à obovales, orbiculaires à la base, arrondies et mucronées au sommet. Les fleurs, blanches ou mauves, sont réunies en d'ammonium, P disponible par la méthode du phosphomolybdate d'ammonium et $K$ par photométrie de flamme (Toth et Prince, 1949).

\subsection{Méthodes de traitement des} données : Les données recueillies sur le sol et les éléments nutritifs de chaque espèce ont été analysées à l'aide du test d'analyse de variance (ANOVA). La différence la moins significative à un niveau de probabilité de $5 \%$ a été utilisée pour faire des comparaisons de traitement.

grappes denses. Le fruit (Fig. 1) est une gousse oblongue, un peu enflée; il est densément et finement tomenteux, vert en début de croissance, brun fauve à gris foncé pendant la phase de déhiscence. Les graines sont suborbiculaires à ovoïdes, de couleur rouge, tachetées de noire. La multiplication se fait par graines.

Phytogéographie et écologie : Espèces pantropicale, Abrus precatorius est rependue dans toute l'Afrique intertropicale où elle pousse dans les formations secondaires. En Côte d'Ivoire, elle a été rencontrée dans les deux aires chronologique, en zones Guinéo-Congolaise et Soudano-Zambézienne.

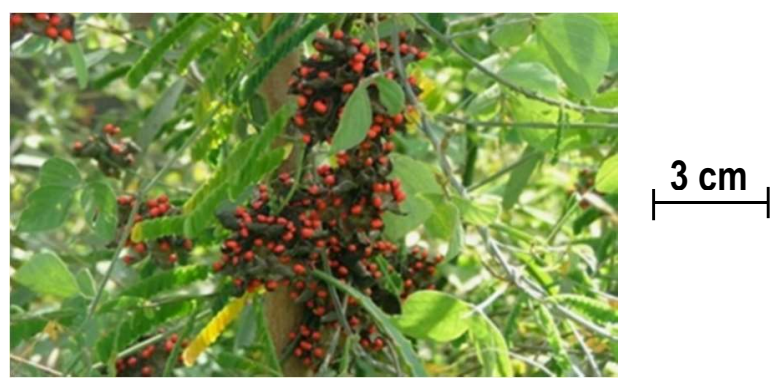

Figure 1: Abrus precatorius L. (Fabaceae). Rameau portant des fruits mûrs laissant entrevoir des graines rouges vifs avec une tâche à la base

\subsubsection{Clitoria ternatea L. (Fabaceae)}

Morphologie : Liane rampante, ses tiges sont grêles puis pubescentes (Fig. 2) Les feuilles sont 
composées imparipennées avec 3 à 7 folioles elliptiques, parfois ovales, finement pubescentes. L'inflorescence est un racème; les fleurs, axillaires, sont solitaires ou géminées et de couleur bleue parfois blanchâtre ou tachetées d'orange au centre. Le fruit est une gousse linéaire oblongue et pubescente, possédant un bec apical. Les graines, noires et réniformes, sont logées dans une alvéole circulaire. Clitoria ternatea se multiplie uniquement par les graines.

Phytogéographie et écologie: Espèce pantropicale, originaire de l'Inde, elle est répandue sous les tropiques et actuellement domestiquée pour l'ornementation ou cultivée dans les jardins.

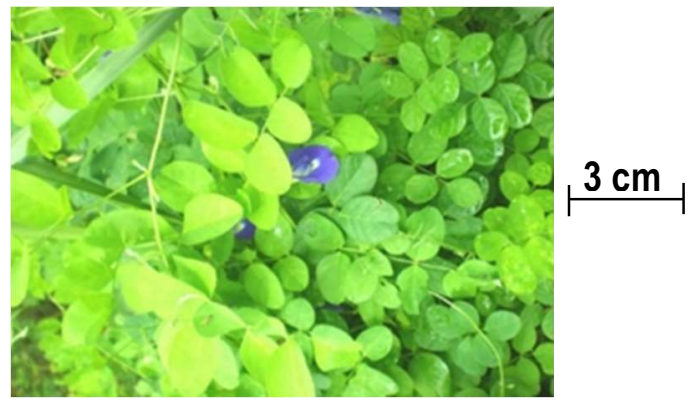

Figure 2 : Clitoria ternatea L. (Fabaceae). Rameaux portant des inflorescences (racèmes) et des fleurs épanouies (couleur bleue)

4.1.1.3 Crotalaria goreensis Guill. et Perr. (Fabaceae)

Morphologie : Herbe à port dressé ou étalé, elle est ramifiée. Les tiges sont côtelés-striés, tomenteux à pubescents, à poils plus ou moins soyeux et étalés. Les feuilles, composées pennées, sont stipulées (Fig. 3). L'inflorescence est un racème terminal, pauci à pluriflore; les fleurs sont jaune- orangé. Le fruit est une gousse sessile, oblongue, ovoïde et pubescente. Les graines sont petites et nombreuses, de teinte rougeâtre. Crotalaria goreensis se reproduit par graines.

Phytogéographie et écologie: Espèce pantropicale, elle est répandue dans toute l'Afrique et pousse quelquefois dans les endroits herbeux, souvent sur les terrains cultivés, dégradés ou dans les jachères.

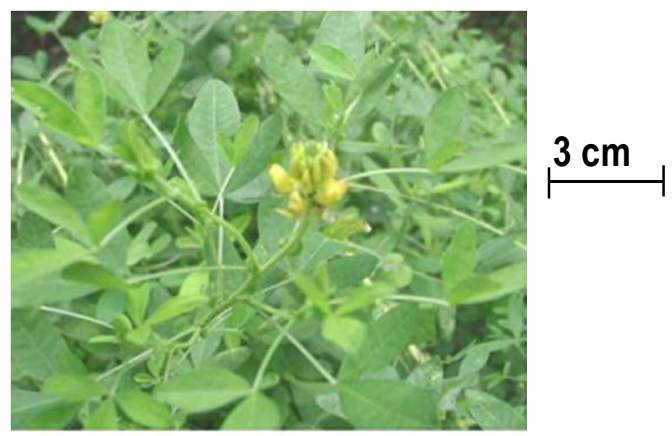

Figure 3 : Crotalaria goreensis Guill. et Perr. (Fabaceae). Rameaux portant une inflorescence (grappe), des fleurs épanouies (couleur jaune), des boutons floraux en formation et des feuilles composées pennées trifoliolées

\subsubsection{Crotalaria retusa L. (Fabaceae)}

Morphologie : Espèce buissonnante, subligneuse, elle peut atteindre $1 \mathrm{~m}$ de hauteur. 
La tige est cylindrique, pleine et faiblement cannelée et couverte d'une pubescence rase. Les feuilles, simples, alternes, sont disposées en spirales assez serrées tout autour de la tige. Le limbe est obovale avec la face supérieure est glabre tandis que la face inferieure est faiblement pubescente. Les fleurs jaunes (Fig. 4), à étendard raté en brun, courtement pédicellées, forment des grappes. Les fruits, des gousses cylindriques terminées par un bec brusquement recourbé, prennent une couleur noire à maturité. Les graines sont lisses et brillantes de couleur brunclair. La plante se multiplie par graines.

Phytogéographie et écologie: Espèce pantropicale, elle est largement répandue dans les zones de foret et savane d'Afrique intertropicale; elle pousse également sur les terrains vagues.
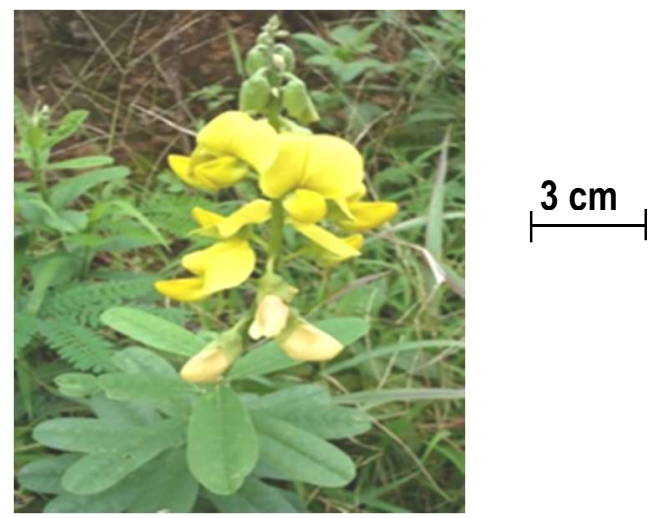

Figure 4 : Crotalaria retusa L. (Fabaceae). Rameaux portant des inflorescences (grappe) et des fleurs épanouies (couleur jaune).

4.1.1.5 Indigofera arrecta Hochst. ex A. Rich. (Fabaceae)

Morphologie : Arbuste vivace, ligneux, à racine pivotante, ses feuilles (Fig. 5), composées paripennées, possèdent des stipules sétacées. Les fleurs, axillaires, subsessiles, rassemblées en inflorescences racémiformes présentent un calice strigueux, une corolle rougeâtre. L'étendard est largement elliptique à subarrondi et sessile. Les fruits sont des gousses étalées, le plus souvent réfléchies, linéaires et parfois glabres. Les graines sont petites de teint noir à maturité. Cette espèce se reproduit par graines. Phytogéographie et écologie: Espèce pantropicale, elle est originaire d'Amérique tropicale ; elle est largement répandue en Afrique de l'Ouest où elle pousse. On la rencontre couramment dans les jachères

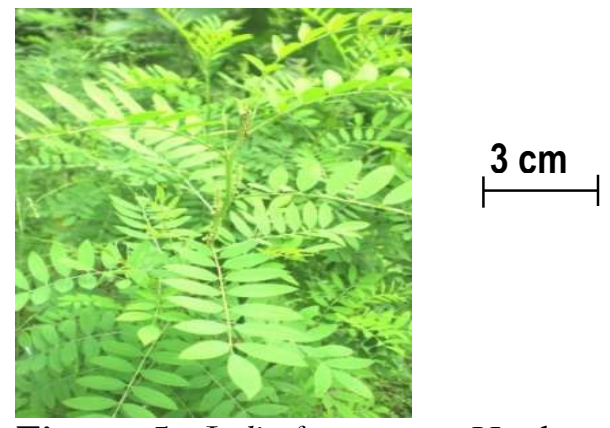

Figure 5: Indigofera arrecta Hochst. ex A. Rich. (Fabaceae). Rameaux portant des inflorescences (grappe), des boutons floraux et des fleurs épanouies .

4.1.2 Espèces de la famille des Morphologie: Herbe plus ou moins vivace, Mimosaceae

4.1.2.1 Mimosa invisa Mart. (Mimosaceae) sarmenteux, la plante comporte des tiges rampantes, parfois dressées. Les feuilles, 
composées bipennées à pétioles et rachis aiguillonnés, possèdent plusieurs paires de pennes ; elles sont très sensitives au toucher ; les pennes, subsessiles, sont linéaires et finement pubescentes. Les fleurs rondes, pédonculées, de couleur rose à pourpre, comportent des pétales longuement soudés. L'inflorescence (Fig. 6) est un capitule axillaire, solitaire. Le fruit est une gousse oblongue et éparsement pubescente. Les graines sont ovales à marge armée de longs poils raides. Mimosa invisa se multiplie par graines.

Phytogéographie et écologie : Espèce pantropicale, elle est originaire d'Amérique tropicale. Elle est considérée comme une mauvaise herbe sous les tropiques. En réalité, c'est une plante de couverture envahissante difficile à extirper lorsqu'elle porte des fruits.

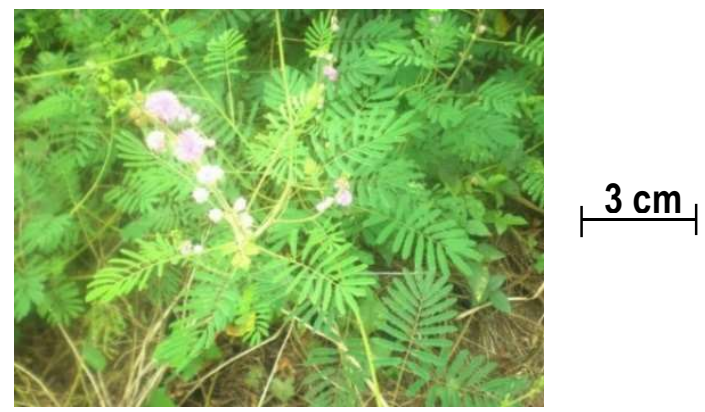

Figure 6: Mimosa invisa Mart. (Mimosaceae). Rameaux portant des inflorescences (capitule), des boutons floraux en formation et des fleurs épanouies (couleur rose).

\subsection{Caractéristisation}

morphologique et biologique des espèces recensées: En rapport avec les Types Biologiques (fig. 7), toutes les espèces recensées ont été des Phanérophytes. Les Microphanérophytes ont été minoritaires (2 espèces soit 33,33\%) contre une majorité de Nanophanérophytes (4 espèces soit 66,67\%). Du point de vue des Types Morphologiques, en fonction de leur taille, la répartition des taxons recensés, au cours de nos investigations, s'est fait en 3 groupes (Fig. 8) : deux espèces d'herbes (soit $33 \%$ ), trois espèces de lianes (soit $50 \%$ ) et une espèce d'arbuste (soit $17 \%$ ). Les lianes ont été donc majoritairement représentées. Certaines, parmi celles-ci, ont été volubiles (Abrus precatorius), d'autres ont été rampantes parfois grimpantes (Clitoria ternatea). Mimosa invisa a été grimpante grâce à ses organes de préhension que sont les épines. La répartition des espèces, par Types Phytogéographiques (Fig. 9), a permis d'établir 4 groupes de plantes. Les taxons de la zone Guinéo-Congolaise (GC) se rencontrent en forêts denses humides, dans le Sud de la Côte-d'Ivoire. Un taxon (soit $17 \%$ ) recensé appartient à cette zone. Un seul taxon (soit $17 \%$ ) de la zone 
Nanophanérophytes $66,67 \%$

\section{Microphanérophytes} $33,33 \%$

Figure 7 : Types Biologiques

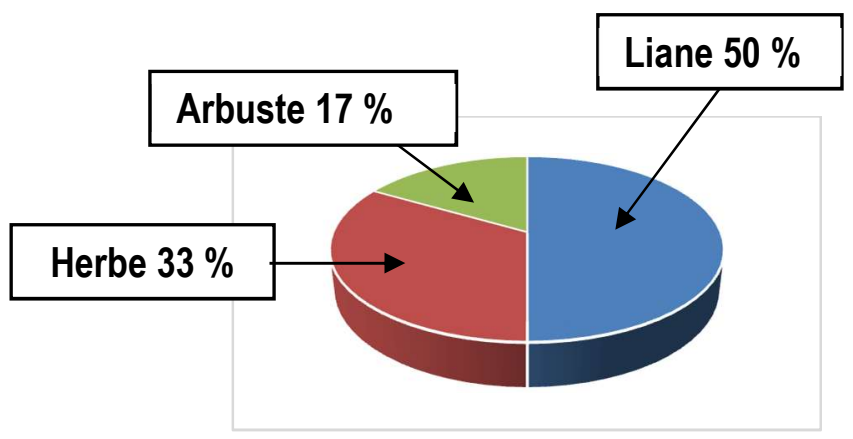

Figure 8 : Types Morphologiques

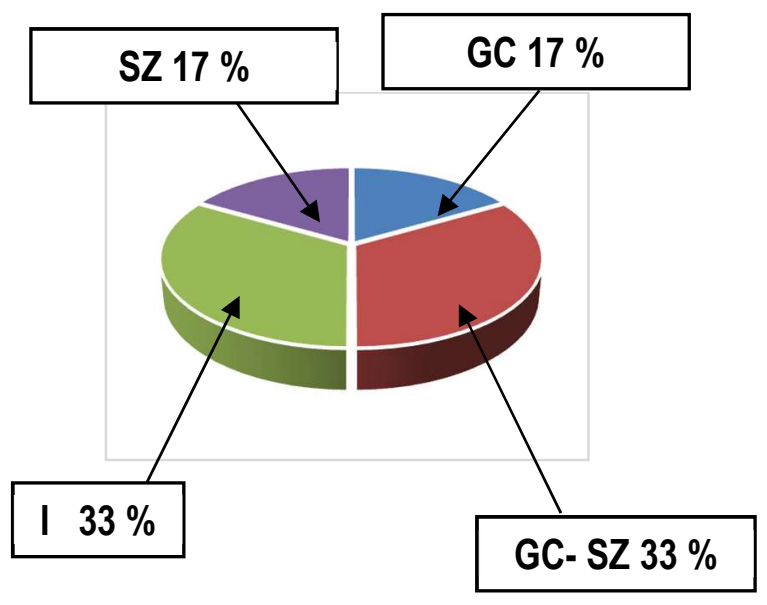

Figure 9 : Types Phytogéographiques

Légende : GuinéoCongolaise-SoudanoZambésienne . (GC-SZ), Guinéo-Congolaise (GC), Introduits (I), Soudano-Zambésienne (SZ)

Soudano-Zambésienne (SZ) a été localisé naturellement, dans la partie septentrionale du pays ; il a été introduit en zone GuinéoCongolaise. Les taxons introduits (I) sont cultivés à diverses fins. Nous en avons recensé deux (02) équivalant à $33 \%$. Des taxons au nombre de 02 (soit $33 \%$ ) ont été communs aux zone Guinéo-Congolaise et SoudanoZambésienne (GC-SZ).

4.3 Caractéristiques chimiques des sols aux pieds des racines de plantes: Des différences significatives ont été observées dans la composition chimique des espèces (tableau I). L'azote a varié de $13 \mathrm{~g} \mathrm{~kg}^{-1}$ (témoin) à $28 \mathrm{~g} \mathrm{~kg}^{-1}$ dans les racines de Mimosa invisa. De plus, les 
concentrations ont été maximales chez Crotalaria goreensis (3,9 $\left.\mathrm{g} \mathrm{kg}^{-1} \mathrm{de} \mathrm{P}\right)$, Crotalaria retusa (53 $\mathrm{g} \mathrm{kg}$ ${ }^{1}$ de $\left.\mathrm{K}\right)$. De plus, les concentrations maximales de CEC (13,8 cmolc kg$\left.^{-1}\right)$ et de C (27 g kg-1) ont été observées avec Mimosa invisa . Pour la plupart des légumineuses (Clitoria ternatea, Abrus precatorius, Indigofera arrecta et Crotalaria goreensis), les concentrations ont été maximales en $\mathrm{N}, \mathrm{P}, \mathrm{K}$. La composition nutritive élevée pour la plupart des légumineuses peut être attribuée au système racinaire étendu de la plante; en présence des légumineuses, la granulométrie du sol a été très significativement améliorée. De la même manière, le $\mathrm{pH}$ du sol s'est relevé progressivement. Cela a plaidé en faveur de l'utilisation de ces légumineuses dans les jachères améliorées.

Tableau 1 : Propriétés physicochimiques du sol sous les légumineuses herbacées ou non (témoin)

\begin{tabular}{|c|c|c|c|c|c|c|c|c|c|}
\hline \multirow{2}{*}{ Espèces } & & \multicolumn{3}{|c|}{ Taille particule (\%) } & \multicolumn{4}{|c|}{$\mathrm{g} \mathrm{kg}^{-1}$} & \multirow{2}{*}{$\begin{array}{c}\begin{array}{c}\text { cmolc } \\
\mathrm{kg}^{-1}\end{array} \\
\text { CEC }\end{array}$} \\
\hline & $\mathrm{pH}$ & argile & limon & sable & $\mathrm{C}$ & $\mathrm{N}$ tot & $P$ disp & K disp & \\
\hline Témoin & $6,3 \pm 1,3$ & $40 \pm 3,0$ & $15,7 \pm 4,0$ & $44,3 \pm 9$ & $1,4 \pm 3,1$ & $13 \pm 1,2$ & $0,8 \pm 0,5$ & $14,4 \pm 1,1$ & $6,1 \pm 3,2$ \\
\hline $\begin{array}{l}\text { A. } \\
\text { preca }\end{array}$ & & $50 \pm 0,2$ & 28 & $21,6 \pm 7$ & $18 \pm 2,2$ & $16 \pm 1,3$ & $=0,1$ & 29 & $6,8 \pm 3,2$ \\
\hline $\begin{array}{l}\text { C. } \\
\text { goreensis }\end{array}$ & $6,4 \pm 2,0$ & $65 \pm 1,1$ & $17,4 \pm 1,2$ & $17,6 \pm 3,1$ & $18 \pm 0,0$ & $14 \pm 1,5$ & $3,9 \pm 0,0$ & $16,8 \pm 1,2$ & $8,6 \pm 2,1$ \\
\hline C. retusa & $, 7 \pm 1,7$ & $50 \pm 0,5$ & $20 \pm 1,3$ & $30 \pm 4,0$ & $19 \pm 1,5$ & $20 \pm 2,0$ & $1,4 \pm 0,0$ & $53,0 \pm 1,1$ & $11,2 \pm 1,8$ \\
\hline C. ternatea & $7,0 \pm 1,2$ & $55 \pm 0,0$ & $19,2 \pm 1,3$ & $25,8 \pm 2,5$ & $23 \pm 2,3$ & $23 \pm 1,1$ & $1,3 \pm 1,2$ & $17,2 \pm 2,0$ & $13,8 \pm 4,0$ \\
\hline I. arrecta & $6,7 \pm 1,4$ & $65 \pm 0,0$ & $22,1 \pm 1,7$ & $12,9 \pm 2,4$ & $26 \pm 1,0$ & $26 \pm 0,0$ & $0,7 \pm 1,2$ & $31,8 \pm 1,0$ & $11,5 \pm 3,4$ \\
\hline M. invisa & $7,0 \pm 1,6$ & $60 \pm 1,0$ & $25,8 \pm 1,4$ & $14,2 \pm 1,9$ & $27 \pm 1,1$ & $28 \pm 1,0$ & $1,3 \pm 0,8$ & $29,7 \pm 1,8$ & $13,8 \pm 2,4$ \\
\hline
\end{tabular}

$\mathrm{N}=4$; les valeurs sont les moyennes de quatre répétitions \pm erreur standard ; disp : disponible

\section{$5 \quad$ DISCUSSION}

Le résultat de l'inventaire a montré que toutes les espèces récoltées appartiennent à deux familles de légumineuses notamment la famille des Fabaceae et celle des Mimosaceae. Ces espèces sont susceptibles de restaurer la fertilité du sol, car elles sont connues pour leur production élevée de la biomasse végétale qui enfouie dans le sol produit de la matière organique. Ainsi, la matière organique fournie par l'apport de la biomasse végétale intervient dans les mécanismes de la libération de l'azote minéral, donc de la nutrition de la culture associée ou subséquente (Gaussen et al., 1982). En sommes, les matières organiques jouent un rôle primordial dans le sol. Elles servent de source d'énergie pour la flore et la faune du sol qui participent à la structure et stabilité structurale du sol. En effet, selon Rouanet (1984), elle influe selon sa nature sur les propriétés physicochimiques et biologiques du sol et a la capacité d'emmagasiner les réserves en eau. Parlant de l'humidité, Bationo et Mokwunye (1991) signalent qu'elle représente un facteur susceptible d'influencer la croissance des cultures et l'utilisation des éléments nutritifs. En dehors de son rôle si important dans le sol, la biomasse végétale enfouie se présente comme un réservoir d'éléments minéraux très variés pour la culture suivante. Selon Rouanet (1984), elle contiendrait au moins cinq (5) éléments minéraux majeurs à savoir l'azote $(\mathrm{N})$, le calcium $(\mathrm{Ca})$, le magnésium $(\mathrm{Mg})$, le potassium $(\mathrm{K})$, le soufre $(\mathrm{S})$ et le phosphore $(\mathrm{P})$ qui, mis à la disposition de la culture associée ou subséquente non fixatrice d'azote, peuvent influencer considérablement sa croissance. Les plantes absorbent des éléments nutritifs du sol sous forme minérale et les transforment en molécules organiques. En effet, la décomposition de ces matières organiques transforment les molécules organiques sous forme minérale, restituant ainsi les éléments nutritifs au sol pour être assimilés par d'autres plantes. Selon Mukendi et Ngoie (2015), elles seraient considérées comme un amendement et 
non pas un engrais à cause de leur impact important sur la structure du sol. Pour cet auteur, la libération progressive des éléments minéraux à partir des biomasses de ces espèces enfouies, pourrait stimuler la croissance des végétaux subséquents pendant une durée de 60 jours. Dès lors, une jachère colonisée par les biofertilisants confirment la disponibilité des éléments minéraux essentiels pour la croissance et le développement des cultures par des apports organo-minérales (Muyayabantu et al., 2012). Selon Persoons et Hassani (1994), la décomposition de ces biofertilisants met en compétition les plantes de culture et les microorganismes du sol pour les nutriments au point que l'azote reste en grande partie immobilisé par les microorganismes. Aussi, ces espèces issues de ces familles ont l'aptitude, par la fixation symbiotique de capturer l'azote atmosphérique qu'elles fixent dans des nodosités situées sur les racines (Morot-Gaudry, 1997). Ce qui leur permettait de solubiliser les phosphores de calcium et le phosphore occlue, augmentant ainsi la disponibilité du phosphore assimilable et son absorption par les cultures associées ou

\section{CONCLUSION}

$\mathrm{Au}$ terme de nos investigations, six espèces de légumineuses ont été identifiées appartenant à deux familles. La famille la plus représentée a été celle des Fabaceae avec cinq espèces qui sont réparties entre quatre genres (Abrus, Clitoria, Crotalaria et Indigofera). La seconde famille a été représentée par les Mimosaceae avec un genre (Mimosa). Toutes ces espèces sont susceptibles de stimuler une dynamique de croissance des cultures subséquentes par le maintien de la fertilité du sol. Ainsi, dans les conditions d'une pression d'exploitation permanente du sol, la plantation dans les jachères améliorées de ces

\section{REFFERENCES BIBLIOGRAPHIQUES}

Akedrin T.N., 2013. Contribution à l'étude de développement de quelques légumineuses herbacées ou subligneuses à utilisation agronomique en Côte d'Ivoire. Thèse unique, Université Félix subséquentes (Serpantié, 1993). D’une part, leur présence permet de lutter contre les adventices pour les cultures associées ou subséquentes. D'autre part, ces espèces jouent un rôle de protection du sol. Part leur grande capacité de production de la biomasse, elles arrivent à couvrir le sol à l'aide des feuilles mortes et le protéger ainsi contre les érosions diverses. Cette analyse est corroborée par les travaux de Toure (2001) qui révèlent que les espèces de ces familles protègent les sols meubles contre diverses érosions (érosion verticale, érosion latérale et érosion par ravinement); même sur les pentes relativement raides, des sols épais de plusieurs mètres peuvent se maintenir. En sommes, quand la matière organique est intégrée au sol, elle confirme l'interaction positive des substrats organiques constatés déjà au niveau de la croissance, comme l'avait aussi remarqué Eden (1993). Au regard des rendements en grains résultants des cultures associées ou subséquentes obtenus sous divers fertilisants indistinctement de leur nature, il se dégage un gain significativement perceptible en poids.

biofertilisants semblent être une alternative intéressante, du fait de leur capacité d'enrichissement rapide des sols par la fixation d'azote atmosphérique et la production abondante de biomasse végétale; ce qui induit la fertilité des sols. Ces résultats constituent un indicateur d'utilisation de légumineuses subligneuses dans les jachères de courte durée à l'effet de contribuer à la promotion de l'apport des fertilisants organiques, organo-minéraux ainsi que minérale utilisé pour améliorer les propriétés physique, chimique et biologique du sol.

Houphouët-Boigny, Côte d'Ivoire. 164 p.

Bado B.V., 2002. Rôle des légumineuses sur la fertilité des sols ferrugineux tropicaux des zones Guinéenne et Soudanienne du Burkina Faso. Ph D., faculté des sciences 
de l'Agriculture et de l'Alimentation. Université de Laval, Québec (Canada), $176 \mathrm{p}$.

Bationo A. et Mokwunye A.U., 1991. Alleviating soil fertility constraints to increased crop production in West Africa: the experience of the Sahel. In: Mokwunye A. Alleviating soil fertility constraints to increased crop production in West Africa. Ed. Kluwer Academic Publisher, Dordrecht, 195-215.

Beedy T. L., Snapp, S. S., Akinnifesi, F. K. and Sileshi, G. W., 2010. Impact of Gliricidia sepium intercropping on soil organic matter fractions in a maize-based cropping system. Agric. Ecosyst. Environ. 38: 139-146

Carsky R. Y.; Singh BB ; OYEMOLE B 2001.Contribution of early season cowpea to late season maize in the Savanna zone of west Africa .Biological Agriculture et Horticulture ; 2001 ; .4p.303-315.

Danso S. K. A., 1995. Assessment of biological nitrogen fixation. Fertilizer Research (42) :33-41.

Eden R., 1993. Effet des fientes de volaille de canaux dans les couloirs de Leuceana leucocephala sur le maïs cultivé sur terre de barre. Thèse d'ingénieur agronome. $73 \mathrm{p}$.

Eneji A. E., Yamamoto S., Wen G., Inanaga S. and Honna, T., 2005. Comparative evaluation of wet digestion and dry ashing methods for the determination of some major and minornutrients in composted manure. Toxicol. Environ. Chem. 87: 147-158

Floret C. et Pontanier R., 2000. La jachère en Afrique tropicale. Rôle, Aménagement, Alternatives. Vol I, Actes de séminaire international, Dakar, 13-16 avril 1999. U.E., CORAF, IRD. Edition John Libbey Eurotext, Paris, 777 pp.

Fournier A., Foret C. et Ghahoua G. M., 2001. Végétation des jachères et succession post culturale en Afrique tropicale. In : C. Floret et G. Serpantié. Symposium international sur la jachère en Afrique tropicale, (Eds.). John Libbey Eurotext. Paris. 2001. La jachère en Afrique tropicale, Synthèse bibliographique. Paris, pp. $123-147$

Gardener W. K., Parbery D. G. et Barber D.A., 2001. Protenoid root morphology and function in Lapinus albus. Plant and soil, (60): 143-147.

Gaussen H., Leroy J. F. et Ozenda P., 1982. Précis de Botanique Tome 2. Végétaux Supérieurs. Edition Masson. 579 p.

Hien et al, 1993.Gestion partagée de l'eau potable à Ouagadougou: pouvoirs urbains et contrôle social. In : Travaux de l'institut ou Géographie de Reims, n.83-84 2e semestre 1993, p 81-97.

Hutchinson J. et Dalziel J. M., 1954. Flora of west Tropical Africa. $2^{\mathrm{e}}$ édition, London, 4 volumes $712 \mathrm{p}$.

Lebrun J. P. et Stork A. L., 1997. Enumération des plantes à fleur d'Afrique Tropicale : Gamopétales: Clethraceae à Lamiaceae. Volume IV: Conservatoire Jardin botanique de la ville de Genève, 712 p.

Morot-Gaudry JF, 1997. Assimilation de l'azote chez les plantes. Aspects physiologique, biochimique et moléculaire. Institut National de la Recherche Agronomique. $254 \mathrm{p}$.

Motsara M. R., and Roy R. N., 2008. Guide to Laboratory establishment for plant nutrient analysis. FAO Fertilizer and Plant nutrition bulletin. Food and Agriculture Organization, Rome. Pp. 219.

Mukendi T.R et Ngoie K.J, 2015. Effet des engrais biologiques sur la production légumière d'amarante (Amaranthus bybridus). Cabiers du Cresa. 179-193.

Muyayabantu G.M, Kadiata B.D and Nkongolo K.K, 2012. Response of maize to different organic and inorganic fertilization regimes in monocrop and intercrop systems in a sub-Saharan Africa region. Journal of Soil Science and Environmental Management, Vol. 3(2) : 4248. 
N'Guessan A. H., N'Guessan. K. F., Kouassi K. P., Kouamé. N. N. et N'Guessan. P.W., 2014. Dynamique des populations du foreur des tiges du cacaoyer, Eulophonotus myrmeleon. Felder (Lépidoptère : Cossidae) dans la région du HautSassandra en Côte d'Ivoire, 9 p.

Partey S. T., Quashie-Sam S. J., Thevathasan N. V. and Gordon, A. M., 2011. Decomposition and nutrient release patterns of the leaf biomass of the wild sunflower (Tithonia diversifolia): acomparative study with four leguminous agroforestry species. Agroforest. Syst. 8, 123-134

Partey S.T., Zougmore R.B., Thevathasan N.V. and Preziosi R.F., 2019. Effects of Plant Residue Decomposition on Soil N Availability, Microbial Biomass and $\beta$ Glucosidase Activity during Soil Fertility Improvement in Ghana. Pedosphere. 29(5): 608-618

Pearsoons C.J. and Hassani A.J., 1994. Maize and pearl millet. In: C.J. Pearson, ed. Control of crop productivity, New York, USA, Academic Press. pp. 141-158.

Pelletier et al, 1995 : Demain d'archimbaud(G), FIXOT(M), Pelletier(T-P), Vallauri(L).Notre Marseille, Ablaye Saint-Victor. Vestiges paléochrétiens .Notice Celle (La), Eglise Notre-Dame de la Gayale In : Duval(N) dir-les premiers monuments chrétiens de la France ,1 Sud-Est et Corse, Paris, 1995, pp.125141,167-174.

Rouanet G, 1984. Le maïs : Technicien d'Agriculture Tropicale. Agence de Coopération Culturelle et Technique. Ed. Maisonneuve et Larousse. 142 p.

Serpantié G., 1993. Rôles et significations de la jachère dans les systèmes de production agricole en Afrique de l'Ouest. Problématique de son remplacement. In : C. Floret et G. Serpantié (Eds.) : pp.55 $-84$.

Toth S. J. and Prince, A. L., 1949. Estimation of cation exchange capacity and exchangeable $\mathrm{Ca}$, Kand $\mathrm{Na}$ contents of soils by flamephotometric techniques. Soil. Sci. 67, 439-445

Toure A., 2001. Etude comparée de l'influence de quatre plantes de couverture sur la flore et la végétation d'une parcelle de jachère de la station de coton du CNRA de Bouaké, Côte d'Ivoire, DEA. de Botanique, Option Ecologie Végétale, Université Cocody-Abidjan (Côte d'Ivoire) U.F.R. Biosciences (Laboratoire de Botanique), 93 p.

Vanhie M., Deen W., Lauzon J. D. and Hooker, D. C., 2015. Effect of increasing levels of maize (Zea mays L.) residue on no-till soybean (Glycine max Merr.) in Northern production regions: A review. Soil Till Res 150: 201-210. 